\title{
Size controlled fabrication of cellulose nanoparticles for drug delivery applications
}

\author{
Suk Fun Chin*, Fiona Beragai Jimmy, Suh Cem Pang \\ Department of Chemistry, Faculty of Resource Science and Technology, Universiti Malaysia Sarawak, 94300, Kota Samarahan, Sarawak, Malaysia
}

\section{A R T I C L E I N F O}

\section{Keywords:}

Cellulose

Nanoparticles

Drug carriers

Size controlled

Nanoprecipitation

\begin{abstract}
A B S T R A C T
Spherically shaped cellulose nanoparticles with average particle diameter range between 70 and $365 \mathrm{~nm}$ were fabricated from cotton fibers by a simple nanoprecipitation method. The average particle diameter of cellulose nanoparticles could be precisely controlled by modulating the synthesis conditions such as cellulose solution concentration and volume ratio of solvent/non-solvent. Methylene blue (MB) as a model hydrophilic drug was loaded onto cellulose nanoparticles via the in-situ nanoprecipitation process. The loading efficiency and release profile of MB were observed to be affected by the average particle diameter of cellulose nanoparticles.
\end{abstract}

\section{Introduction}

Natural biopolymers or polysaccharides (e.g., starch, cellulose, and gelatin) are gaining popularity as the precursor materials for the fabrication of nanoparticles because they are non-toxic, biodegradable, biocompatible and low cost [1]. Among these biopolymers, cellulose is of particular interest as it is abundantly available, renewable, and can be isolated from various natural resources such as wood, cotton, wheat straw, coconut husk, sugarcane bagasse, sesame husk, hemp fiber, and banana rachis [2].

Cellulose-based nanoparticles have great potential in various applications such as personal cares, foods, electronic and pharmaceuticals due to their distinctive properties. However, researches on the fabrication of cellulose nanoparticles are relatively scarce as compared to others polysaccharide-based nanoparticles such as chitosan, alginate and dextran. Some researchers have attempted to fabricate cellulose nanoparticles through hydrolysis of cellulose with concentrated sulfuric acid $\left(\mathrm{H}_{2} \mathrm{SO}_{4}\right)$. Cellulose nanoparticles with average particle diameter ranging from 30 to $120 \mathrm{~nm}$ were produced from an agro-waste (sesame husk) through the acid hydrolysis process [3]. However, $\mathrm{H}_{2} \mathrm{SO}_{4}$ is a strong acid, and a very corrosive reagent, thus required special processing reactors. As such the synthesis method is not promising for the large-scale fabrication of cellulose nanoparticles [4]. Furthermore, this method does not afford precise control of the particle diameters of cellulose nanoparticles.

The nanoprecipitation method was explored by several researchers for the fabrication of polymeric nanoparticles due to its simplicity and reproducibility $[5,6]$. Herein, we have reported on the fabrication of cellulose nanoparticles with controllable average particle diameter from cotton fibers using the nanoprecipitation method. Cellulose nanoparticles of average particle diameter ranging from 70 to $365 \mathrm{~nm}$ were obtained through optimizing the synthesis conditions such as ratio of solvent/non-solvent, concentrations of the cellulose, and the use of water-in-oil (w/o) microemulsion. The potential application of these cellulose nanoparticles as controlled release carriers was being explored by using methylene blue (MB) as a model hydrophilic drug. The effects of average particle diameter of cellulose nanoparticles on drug loading efficiency and release kinetic profile were investigated.

\section{Materials and method}

\subsection{Materials}

Facial cotton was purchased from Watson Personal Care Stores Sdn. Bhd., Malaysia. Sodium hydroxide $(\mathrm{NaOH})$, thiourea, polysorbate- 80 (Tween-80) surfactant, and Methylene Blue (MB) were obtained from Merck (Germany), cyclohexane, hydrochloric acid ( $\mathrm{HCl})(37 \%)$ and absolute ethanol were obtained from HmbG Chemicals (Germany) and urea was obtained from UNI-CHEM (India). All chemicals were used without further purification. Ultrapure water $\left(\sim 18.2 \mathrm{M} \Omega . \mathrm{cm}, 25{ }^{\circ} \mathrm{C}\right)$ was obtained from the Water Purifying System (ELGA, Model Ultra Genetic).

\subsection{Chemical pretreatment}

Facial cotton as the cellulose source, was cut into smaller pieces and then soaked in $\mathrm{NaOH}$ solution ( $12 \mathrm{w} / \mathrm{v} \%$ ) for $2 \mathrm{~h}$ followed by sonication [7]. The resulting slurry was subjected to acid treatment with $1 \mathrm{M}$

\footnotetext{
* Corresponding author.

E-mail address: sukfunchin@gmail.com (S.F. Chin).
} 